\title{
A NOTE ON BOUNDARY REGULARITY OF SUBELLIPTIC HARMONIC MAPS*
}

\author{
ZHEN-RoNG ZHOU
}

\begin{abstract}
Let $M^{m}=\Omega$ be a bounded domain of $\mathbf{R}^{m}$. Jost and $\mathrm{Xu}$ in [7] introduced the subelliptic harmonic map from it and proved the regularity up to the boundary of subelliptic harmonic maps into small balls supposing that the boundary of the domain is noncharacteristic and smooth. In this note, we investigate the boundary regularity of this kind of maps from $\Omega$ with non-smooth and partial noncharacteristic boundary.
\end{abstract}

\section{Introduction, main result}

Let $M$ and $N$ be two Riemannian manifolds. A critical point in Sobolev space $H^{1}(M, N)$ of the energy functional is called a (weakly) harmonic map. Denote a geodesic ball with radius $R$ and center $p \in N$ by $B_{R}(p) . \quad B_{R}(p)$ is said to be small, if $R<\min \left\{\frac{\pi}{2 \sqrt{\kappa}}, i(p)\right\}$ where $\kappa$ is a positive upper bound of the sectional curvature of $N$ and $i(p)$ is the injective radius of $p$. For instance, any ball of the Euclidean space is small, and so is any geodesic ball with radius less than $\pi / 2$ in unit spheres. Hildebrandt et al. proved that harmonic maps into a small ball are regular up to the boundary (see [6]).

Let $M^{m}=\Omega$ be a bounded domain of $\mathbf{R}^{m}$. Jost and $\mathrm{Xu}$ in [7] introduced the subelliptic harmonic map (see the next section), which is a variant of the classical harmonic map, and proved the regularity up to the boundary of subelliptic harmonic maps into small balls supposing that the boundary of the domain is noncharacteristic and smooth. In this note, we investigate the boundary regularity of this kind of maps from $\Omega$ with non-smooth and partial noncharacteristic boundary.

We call a vector $v$ a outward normal to $\partial \Omega$ at $x \in \partial \Omega$, if there is an Euclidean ball $B$ with center $x_{0}$, such that $\bar{B} \cap \bar{\Omega}=\{x\}$ and $v=k\left(x_{0}-x\right)$ for some positive number $k$. Apparently, if $x$ is a smooth point of the boundary, $v$ is the usual outward normal vector.

\footnotetext{
* Research supported by the Natural Science Foundation of China No. 10371047 and No. 10571068 .

Received August 16, 2004; revised March 7, 2005.
} 
Let $X=\left\{X_{1}, \ldots, X_{k_{0}}\right\}$ be a family of Hörmander's vectors, and $\Delta_{X}$ be the subLaplacian defined by $X$ (see next section). Write the terms of the second order of $-\Delta_{X}$ by $\sum_{i, j=1}^{m} a_{i j} \frac{\partial^{2}}{\partial x_{i} \partial x_{j}}$. Let $x \in \partial \Omega$. If there exists a outward normal vector $v=\left(v^{1}, \ldots, v_{m}\right)$ at $x$ to $\partial \Omega$ such that $\sum_{i, j=1}^{m} a_{i j} v^{i} v^{j}>0, x$ is called a noncharacteristic point of $\partial \Omega$ with respect to $X$. The set of all noncharacteristic points is denoted by $S_{1}$.

Our main theorem is

Theorem. Suppose that $u: \Omega \rightarrow B_{R}(p) \subset N$ is subelliptic harmonic, where $B_{R}(p)$ is a small ball. If $u=\phi$ on $\partial \Omega$ where $\phi \in C^{2}\left(\Omega \cup S_{1}, B_{R}(p)\right)$, then $u$ is continuous to $S_{1}$.

The plan of this paper is: in Section 2, we collect some useful definitions and facts; in Section 3, we prove the main theorem. In the last two sections, we proof two lemmas which are used in the proof of the main theorem.

\section{Preliminaries}

Let $\Omega$ be an open domain in $\mathbf{R}^{m}, X=\left(X_{1}, X_{2}, \ldots, X_{k_{0}}\right)$ be a family of $C^{\infty}$ vector fields on $\Omega$. We call $X_{\alpha}$ to be Hömander's vector fields if they satisfy the following Hörmander's condition:

$X_{a}, \ldots, X_{k_{0}}$ together with their commutators up to a fixed length $r$ span the tangent space at each point of $\Omega$.

For any collection of vector fields $X=\left(X_{\alpha}, \alpha=1, \ldots, k_{0}\right)$, we define an associated operator of second order as follows:

$$
\Delta_{X}:=\sum X_{\alpha}^{*} X_{\alpha}
$$

where, $X_{\alpha}^{*}$ is the adjoint operator of $X_{\alpha}$ with respect to the Lebesgue's measure. If $X$ satisfies Hörmander's condition, the operator $\Delta_{X}$ is hypeoelliptic, i.e. if $\Delta_{X} u$ is smooth, then so is $u$. In this case, the operator is called Hömander's operator, or called a subelliptic Laplacian or a sub-Laplacian. In general, this is a degenerated elliptic operator.

Let $N$ be a Riemannian manifold with Riemannian metric $g$. Without loss of generality, we assume that $N$ is a submanifold of $\mathbf{R}^{v}$ by Nash's embedding theorem. Define

$$
M^{1, p}\left(\Omega, \mathbf{R}^{v}\right):=\left\{u: \Omega \rightarrow \mathbf{R}^{v} \mid u \in L^{p} ; X_{\alpha} u \in L^{p}, \alpha=1, \ldots, k_{0}\right\}
$$

and define $M_{0}^{1, p}\left(\Omega, \mathbf{R}^{v}\right)$ to be the closure of $C_{0}^{\infty}\left(\Omega, \mathbf{R}^{v}\right)$ in $M^{1, p}\left(\Omega, \mathbf{R}^{v}\right)$ with respect to the norm

$$
\|u\|_{M^{1, p}}^{p}:=\int_{\Omega}|u|^{p} \mathrm{~d} x+\int_{\Omega} \sum\left|X_{\alpha} u\right|^{p} \mathrm{~d} x
$$




$$
\begin{aligned}
& M^{1}(\Omega, N):=\left\{u \in M^{1,2}\left(\Omega, \mathbf{R}^{v}\right): u(\Omega) \subset N \text { a.e. }\right\} . \\
& M_{0}^{1}(\Omega, N):=\left\{u \in M_{0}^{1,2}\left(\Omega, \mathbf{R}^{v}\right): u(\Omega) \subset N \text { a.e. }\right\} .
\end{aligned}
$$

Let $u$ be in $M^{1}(\Omega, N)$ whose coordinate representation is also denoted by $u$. Define the energy density of $u$ by

$$
e(u)(x)=\frac{1}{2} \sum g_{i j}(u(x)) X_{\alpha} u^{i}(x) X_{\alpha} u^{j}(x)
$$

and the energy by $E(u)=\int_{\Omega} e(u) \mathrm{d} x$.

A critical point of the energy functional is called a subelliptic harmonic map. The Euler-Lagrange equations of the energy functional reads (see [11])

$$
\tau(u)=: \sum X_{\alpha}^{*} X_{\alpha} u^{l}-\sum \Gamma_{i k}^{l} X_{\alpha} u^{i} X_{\alpha} u^{k}=0 .
$$

When $N$ is the Euclidean line, the above system is linear, and its solutions are called subelliptic functions. We have

Lemma 1. Suppose that $\Omega \subset \mathbf{R}^{n}$ is a bounded domain. If $g \in$ $M^{1} \cap L^{\infty}(\Omega, \mathbf{R})$ satisfies

$$
\begin{aligned}
\Delta_{X} g \leq 0 & \text { in } \Omega \\
g \leq 0 & \text { on } \partial \Omega
\end{aligned}
$$

Then, $g \leq 0$ in $\bar{\Omega}$.

Proof. Let $g^{+}=\max (g, 0)$. Then by hypothesis, $g^{+} \in M_{0}^{1}(\Omega, \mathbf{R})$. So we can use it as a test function and obtain

$$
\begin{aligned}
\int_{\Omega} \Delta_{X} g \cdot g^{+} \mathrm{d} x & =\int_{\Omega} X_{\alpha} g^{+} X_{\alpha} g^{+} \mathrm{d} x \\
& \leq 0 .
\end{aligned}
$$

Hence we have $X_{\alpha} g^{+}=0$ on $\Omega$ for any $\alpha=1,2, \ldots, k_{0}$. Hence $\left[X_{\alpha}, X_{\beta}\right] g^{+}=$ $\left(X_{\alpha} X_{\beta}-X_{\beta} X_{\alpha}\right) g^{+}=0, \quad\left[\left[X_{\alpha}, X_{\beta}\right], X_{\gamma}\right] g^{+}=\left(\left[X_{\alpha}, X_{\beta}\right] X_{\gamma}-X_{\gamma}\left[X_{\alpha}, X_{\beta}\right]\right) g^{+}=0, \ldots$ By Hörmander's condition we have $X g^{+}=0$ for any tangent vector field $X$ on $\Omega$. Therefore $g^{+}=$const. whence $g^{+}=0$ because $\left.g^{+}\right|_{\partial \Omega}=0$.

Q.E.D.

\section{Proof of the main theorem}

Let $x_{0} \in \partial \Omega$. Denote $f$ be a convex function over $\overline{B_{R}(p)} \subset N$ with unique minimum 0 at $u\left(x_{0}\right) \in B_{R}(p)$. Such a function exists if $B_{R}(p)$ is a small ball. For example, we can take

$$
f(x)=\frac{1-\cos \left(\sqrt{\kappa} \operatorname{dist}\left(u\left(x_{0}\right), x\right)\right)}{\kappa \cos (\sqrt{\kappa} \operatorname{dist}(p, x))} .
$$


The proof of the convexity of $f$ is arranged in the last section (see Lemma 3 ). Note that $\operatorname{dist}^{2}(p, x)$ is convex in regular ball $\overline{B_{R}(p)}$, but $u\left(x_{0}\right)$ is not its minimum, and that $\operatorname{dist}\left(u\left(x_{0}\right), x\right)$ has unique minimum 0 at $u\left(x_{0}\right)$, but it may not be convex in small ball $\overline{B_{R}(p)}$ because $\operatorname{dist}\left(u\left(x_{0}\right), x\right)$ may be bigger than $\frac{\pi}{2 \sqrt{\kappa}}$. If we let $\kappa$ tend to zero, then $f(x)$ tend to $\frac{1}{2}\left(\operatorname{dist}\left(u\left(x_{0}\right), x\right)\right)^{2}$. Hence we call this function the modified distance. Let $g$ be a bounded solution of the following problem:

$$
\begin{aligned}
\Delta_{X} g & =0 & & \text { in } \Omega \\
g & =f \circ u & & \text { on } \partial \Omega .
\end{aligned}
$$

The solvability of this problem is proved in Section 4 (see Lemma 2). The chain rule is (see [7] or [11])

$$
-\Delta_{X}(f \circ u)=\sum \operatorname{Hess} f\left(X_{\alpha} u, X_{\alpha} u\right)-\langle\operatorname{grad} f, \tau(u)\rangle .
$$

Because $u$ is subelliptic harmonic and $f$ is convex, we have $-\Delta_{X}(f \circ u) \geq 0$ by the chain rule. So

$$
-\Delta_{X}(f \circ u-g) \geq 0 \text {. }
$$

But (recall $\phi$ is the boundary value of $u$ )

$$
\left.(f \circ u-g)\right|_{\partial \Omega}=\left.(f \circ \phi-f \circ \phi)\right|_{\partial \Omega}=0
$$

which combined with Eq. (12) implies

$$
f \circ u-g \leq 0
$$

in $\Omega$ by Lemma 1. Hence we have

$$
\begin{aligned}
0 & \geq f(u(x))-g(x) \\
& =f(u(x))-f\left(\phi\left(x_{0}\right)\right)+f\left(\phi\left(x_{0}\right)\right)-g(x) \\
& =f(u(x))-f\left(\phi\left(x_{0}\right)\right)+g\left(x_{0}\right)-g(x) \\
& =f(u(x))+g\left(x_{0}\right)-g(x)
\end{aligned}
$$

because $f\left(\phi\left(x_{0}\right)\right)=f\left(u\left(x_{0}\right)\right)=0$ by the definition of $f$. Hence we get

$$
f(u(x)) \leq g(x)-g\left(x_{0}\right)
$$

On the other hand, by Taylor's expansion and the convexity of $f$ (Lemma 3), we have

$$
\begin{aligned}
f(u(x)) & =\frac{1}{2} \operatorname{Hess} f(\xi)(h, h) \quad \text { where } h=\exp _{u\left(x_{0}\right)}^{-1}(u(x)) \\
& \geq \frac{1}{2} \lambda|h|^{2} \\
& =\frac{1}{2} \lambda \operatorname{dist}\left(u(x), u\left(x_{0}\right)\right)^{2}
\end{aligned}
$$


where Hess $f$ is the Hessian of $f, \lambda$ is the infimum of the smallest eigenvalue of Hess $f$ on $B_{R}(p)$, and $\xi \in B_{|h|}\left(u\left(x_{0}\right)\right)$. So

$$
\operatorname{dist}\left(u(x), u\left(x_{0}\right)\right)<\sqrt{\frac{2}{\lambda}\left(g(x)-w\left(g_{0}\right)\right)} .
$$

If $x_{0} \in S_{1}$, then $g$ is continuous to $x_{0}$ by Lemma 2. Letting $x$ tend to $x_{0}$, we have $\operatorname{dist}\left(u(x), u\left(x_{0}\right)\right) \rightarrow 0$, since $g(x) \rightarrow g\left(x_{0}\right)=f\left(u\left(x_{0}\right)\right)=0$.

Q.E.D.

\section{Linear boundary valued problem}

In this section, we solve the linear boundary valued problem which is used in Section 3. We have

Lemma 2. Let $S_{1}$ be the noncharacteristic part of the boundary of $\Omega$ which satisfies the exterior ball condition. Suppose that $\phi \in C^{2}\left(\Omega \cup S_{1}, \mathbf{R}\right)$. Then there exists a bounded solution $u$ to the following linear boundary valued problem:

$$
\begin{cases}\Delta_{X} u=0 & \text { in } \Omega \\ u=\phi & \text { on } \partial \Omega .\end{cases}
$$

The solution $u$ is smooth in $\Omega$ and continuous to $S_{1}$.

Proof. Denote

$$
\mathscr{B}_{R}(\phi)=\left\{u \in M^{1}(\Omega ; N) \mid u-\phi \in M_{0}^{1}(\Omega ; N) \text { and } \operatorname{dist}(u(x), p) \leq R\right\} .
$$

Let $R<R^{\prime}<\min \{i(p), \pi / 2 \sqrt{\kappa}\}$. Then, we have ([7]):

Let $u \in \mathscr{B}_{R^{\prime}}(\phi)$ satisfy $E(u)=\inf _{v \in \mathscr{B}_{R^{\prime}}(\phi)} E(v)$. If $\phi(\Omega) \subset B_{R}(p)$,

then, $u \in \mathscr{B}_{R}(\phi)$, and $u$ is weakly subelliptic harmonic.

If $N=\mathbf{R}$, the one dimensional Euclidean space, the above fact implies the existence of bounded weak solutions of (19).

Because $\Delta_{X}$ is hypoelliptic, $u$ is smooth in $\Omega$. In the following, we discuss the boundary regularity of $u$. Let $v=u-\phi$. Then $v$ solves the boundary valued problem

$$
\begin{cases}\Delta_{X} v=-\Delta_{X} \phi & \text { in } \Omega \\ v=0 & \text { on } \partial \Omega .\end{cases}
$$

Write $\Delta_{X}$ as $-\sum_{i, j=1}^{m} a_{i j} \frac{\partial^{2}}{\partial x^{i} \partial x^{j}}+\sum_{j=1}^{m} b_{j} \frac{\partial}{\partial x^{j}}$. Let $x_{1} \in S_{1}$ and let $v=\left(v^{i}\right)$ be a outward normal vector to $\partial \Omega$ at $x_{1}$ such that $\sum a_{i j} v^{i} v^{j}>0$. Let $B_{r}\left(x_{0}\right)$ be the Euclidean ball centered at $x_{0}$ such that $\overline{B_{r}\left(x_{0}\right)} \cap \bar{\Omega}=x_{1}$, and that $v=k\left(x_{0}-x_{1}\right)$ for some positive number $k$. Let $h(x)=e^{-k\left|x-x_{0}\right|^{2}}-e^{-k\left|x_{0}-x_{1}\right|^{2}}$, where $|x|$ denotes the Euclidean norm of $x$. It is easily to check $h(x)<0$ for all $x \in \bar{\Omega} \backslash\left\{x_{1}\right\}$ and $h\left(x_{1}\right)=0$. Then we have 


$$
\begin{aligned}
-\Delta_{X} h\left(x_{1}\right) & =\left(4 k^{2} \sum_{i, j=1}^{m} a_{i j}\left(x_{1}^{j}-x_{0}^{j}\right)\left(x_{1}^{i}-x_{0}^{i}\right)+\text { lower terms of } k\right) e^{-k\left|x_{1}-x_{0}\right|^{2}} \\
& \geq 2 c
\end{aligned}
$$

where $c$ is a positive constant, if we let $k$ large enough. Hence there is a neighborhood $U$ of $x_{1}$ such that $-\Delta_{X} h(x) \geq c>0$ in $U$. Let $V=U \cap \Omega$ and $\partial V=B_{1} \cup B_{2}$, where $B_{1}=\partial \Omega \cap \partial V$ and $B_{2}=\partial V \backslash B_{1}$. Then $\left.v\right|_{B_{1}}=0,\left.h\right|_{B_{1}} \leq 0$ and $\left.h\right|_{B_{2}}<-\varepsilon$ for some small positive number $\varepsilon$. Choose a positive number $M$ large enough, we have (note that $\Delta \phi$ is bounded on $\Omega \cup S_{1}$, because $\phi \in C^{2}\left(\Omega \cup S_{1}, \mathbf{R}\right)$. $)$

$$
\begin{aligned}
-\Delta_{X}(M h \pm v) & \geq M c \mp \Delta_{X} \phi \geq 0 & & \text { in } V \\
(M h \pm v) & \leq 0 & & \text { on } \partial V .
\end{aligned}
$$

By Lemma 1 and also noting $h \leq 0$, we reach $\pm v \leq-M h=M|h|$. Thus

$$
|v| \leq M|h| \text { in } V \text {. }
$$

Because $h(x) \rightarrow h\left(x_{1}\right)=0$ as $x \rightarrow x_{1}$, we have $v(x) \rightarrow 0=v\left(x_{1}\right)$. Therefore, $v$ is continuous at $x_{1}$ and hence $u$.

Q.E.D.

\section{Convexity of the modified distance}

Let $N^{n}$ be a Riemannian manifold, the sectional curvature of which is bounded above by a positive number $\kappa$. Let $p \in N$ and $B_{R}(p)$ be a regular ball. For a fixed point $q \in B_{R}(p)$, define

$$
f(x)=\frac{1-\cos (\sqrt{\kappa} \operatorname{dist}(q, x))}{\kappa \cos (\sqrt{\kappa} \operatorname{dist}(p, x))} .
$$

In this section, we prove the following lemma:

Lemma 3. Hess $f(v, v) \geq \lambda|v|^{2}$ on $B_{R}(p)$, where $\lambda$ is a positive number, $v$ is any tangent vector at the point under consideration.

Proof. The following calculation is at $x \in B_{R}(p)$. Let $\Phi$ be a smooth function on $B_{R}(p), \nabla$ the Riemannian connection, grad the gradient operator. Then

$$
\begin{gathered}
\operatorname{grad} e^{\Phi}=e^{\Phi} \operatorname{grad} \Phi \\
\nabla_{v} \operatorname{grad} e^{\Phi}=e^{\Phi}\langle\operatorname{grad} \Phi, v\rangle+e^{\Phi} \nabla_{v} \operatorname{grad} \Phi
\end{gathered}
$$

Hence

$$
\text { Hess } e^{\Phi}(v, v)=e^{\Phi}\langle\operatorname{grad} \Phi, v\rangle^{2}+e^{\Phi} \operatorname{Hess} \Phi(v, v) .
$$

Let $w$ be a smooth function on $B_{R}(p)$. By a straightforward calculation, we have 


$$
\begin{aligned}
\operatorname{Hess}\left(e^{\Phi} w\right)= & w \operatorname{Hess} e^{\Phi}(v, v)+e^{\Phi} \operatorname{Hess} w(v, v) \\
& +2\left\langle\operatorname{grad} e^{\Phi}, v\right\rangle\langle\operatorname{grad} w, v\rangle .
\end{aligned}
$$

Inserting (25) and (27) into (28) yields

$$
\begin{aligned}
e^{-\Phi} \operatorname{Hess}\left(e^{\Phi} w\right)(v, v)= & \text { Hess } w(v, v)+w \operatorname{Hess} \Phi(v, v)+w\langle\operatorname{grad} \Phi, v\rangle^{2} \\
& +2\langle\operatorname{grad} \Phi, v\rangle\langle\operatorname{grad} w, v\rangle .
\end{aligned}
$$

Let $\Phi=\omega \circ \psi$, where $\psi: B_{R}(p) \rightarrow \mathbf{R}$ and $\omega: \mathbf{R} \rightarrow \mathbf{R}$. Then

$$
\operatorname{grad} \Phi=\dot{\omega} \circ \psi \operatorname{grad} \psi,
$$

$$
\text { Hess } \Phi(v, v)=\ddot{\omega} \circ \psi\langle\operatorname{grad} \psi, v\rangle^{2}+\dot{\omega} \circ \psi \operatorname{Hess} \psi(v, v) \text {. }
$$

Substituting (30) and (31) into (29), we have

$$
\begin{aligned}
e^{-\Phi} \operatorname{Hess}\left(e^{\Phi} w\right)(v, v) & \\
= & \operatorname{Hess} w(v, v)+w\left(\ddot{\omega} \circ \psi\langle\operatorname{grad} \psi, v\rangle^{2}+\dot{\omega} \circ \psi \operatorname{Hess} \psi(v, v)\right) \\
& +w(\dot{\omega} \circ \psi)^{2}\langle\operatorname{grad} \psi, v\rangle^{2}+2 \dot{\omega} \circ \psi\langle\operatorname{grad} \psi, v\rangle\langle\operatorname{grad} w, v\rangle .
\end{aligned}
$$

If $\ddot{\omega}=\dot{\omega}^{2}$, then

$$
\begin{aligned}
e^{-\Phi} \operatorname{Hess}\left(e^{\Phi} w\right)(v, v) \\
=\operatorname{Hess} w(v, v)+2 w(\dot{\omega} \circ \psi)^{2}\langle\operatorname{grad} \psi, v\rangle^{2}+w \dot{\omega} \circ \psi \text { Hess } \psi(v, v) \\
\quad+2 \dot{\omega} \circ \psi\langle\operatorname{grad} \psi, v\rangle\langle\operatorname{grad} w, v\rangle .
\end{aligned}
$$

Let $\quad w>0 . \quad$ By Young's inequality: $a b \leq \frac{1}{2} t a^{2}+\frac{1}{2 t} b^{2} \quad$ with $a=-2 \dot{\omega}$ 。 $\psi\langle\operatorname{grad} \psi, v\rangle, b=\langle\operatorname{grad} w, v\rangle$, and $t=w$, we have

$$
-2 \dot{\omega} \circ \psi\langle\operatorname{grad} \psi, v\rangle\langle\operatorname{grad} w, v\rangle \leq 2 w(\dot{\omega} \circ \psi)^{2}\langle\operatorname{grad} \psi, v\rangle^{2}+\frac{1}{2 w}\langle\operatorname{grad} w, v\rangle^{2} .
$$

Applying (34) to (33) we have

$$
\begin{aligned}
e^{-\Phi} \operatorname{Hess}\left(e^{\Phi} w\right)(v, v) \geq & \operatorname{Hess} w(v, v)+w \dot{\omega} \circ \psi \operatorname{Hess} \psi(v, v) \\
& -\frac{1}{2 w}\langle\operatorname{grad} w, v\rangle^{2} .
\end{aligned}
$$

Set $c_{\kappa}(t)=\cos (\sqrt{\kappa} t), s_{\kappa}(t)=\frac{1}{\sqrt{\kappa}} \sin (\sqrt{\kappa} t)$. It is easily to get $\dot{c}_{\kappa}=-\kappa s_{\kappa}, \dot{s}_{\kappa}=c_{\kappa}$, and $\kappa s_{\kappa}^{2}+c_{\kappa}^{2}=1$. Let $r_{q}(x)=\operatorname{dist}(q, x), q_{\kappa}(t)=\frac{1}{\kappa}\left(1-c_{\kappa}(t)\right)$, and $w=q_{\kappa} \circ r_{q}$.

$$
\operatorname{grad} w=s_{\kappa} \circ r_{q} \operatorname{grad} r_{q}
$$


Furthermore, if $x \neq q$ we have $w>0$. By a straightforward calculation, one has

$$
\text { Hess } w(v, v)=\dot{s}_{\kappa} \circ r_{q}\left\langle\operatorname{grad} r_{q}, v\right\rangle^{2}+s_{\kappa} \circ r_{q} \text { Hess } r_{q}(v, v) .
$$

Let $\gamma$ be the minimizing geodesic from $\gamma(0)=q$ to $\gamma(\rho)=x$, where $\rho=r_{q}(x)$. Denote $v^{\top}=\langle v, \dot{\gamma}(\rho)\rangle \dot{\gamma}(\rho), v^{\perp}=v-v^{\top}$. By [6] (p. 59), we have (it is sufficient to take $v_{1}=0$ and $v_{2}=v$ )

$$
\text { Hess } r_{q}(v, v) \geq \frac{\dot{s}_{\kappa} \circ r_{q}}{s_{\kappa} \circ r_{q}}\left|v^{\perp}\right|^{2} .
$$

Apply (38) to (37). We have

$$
\text { Hess } \begin{aligned}
w(v, v) & \geq \dot{s}_{\kappa} \circ r_{q}\left\langle\operatorname{grad} r_{q}, v\right\rangle^{2}+\dot{s}_{\kappa} \circ r_{q}\left|v^{\perp}\right|^{2} \\
& =\dot{s}_{\kappa} \circ r_{q}\left|v^{\top}\right|^{2}+\dot{s}_{\kappa} \circ r_{q}\left|v^{\perp}\right|^{2} \\
& =\dot{s}_{\kappa} \circ r_{q}|v|^{2} \\
& =(1-\kappa w)|v|^{2} .
\end{aligned}
$$

Let $\psi=q_{\kappa} \circ r_{p}$. Then by (39) we have

$$
\text { Hess } \psi(v, v) \geq(1-\kappa \psi)|v|^{2} \text {. }
$$

Take $\omega(t)=-\log (1-\kappa t)$ and notice that $0<\kappa \psi<1$ since $x \neq q$ and $r_{p}(x)<\frac{1}{2 \sqrt{\kappa}}$. Then $\dot{\omega}(t)=\kappa /(1-\kappa t), \ddot{\omega}=\dot{\omega}^{2}$. Taking use of (39) and (40) in $(35)$, we get

$$
\begin{aligned}
e^{-\Phi} & \operatorname{Hess}\left(e^{\Phi} w\right)(v, v) \\
& \geq(1-\kappa w)|v|^{2}+w \dot{\omega} \circ \psi(1-\kappa \psi)|v|^{2}-\frac{1}{2 w}\langle\operatorname{grad} w, v\rangle^{2} \\
& =(1-\kappa w)|v|^{2}+\kappa w|v|^{2}-\frac{1}{2 w}\langle\operatorname{grad} w, v\rangle^{2} \\
& =|v|^{2}-\frac{1}{2 w}\left(s_{\kappa} \circ r_{q}\right)^{2}\left\langle\operatorname{grad} r_{q}, v\right\rangle^{2} \\
& =|v|^{2}-\frac{1}{2}\left(1+c_{\kappa} \circ r_{q}\right)\left\langle\operatorname{grad} r_{q}, v\right\rangle^{2} \\
& >0 \quad \text { if } x \neq q \text { and } v \neq 0 .
\end{aligned}
$$

At $x=q, w=0$ and $\operatorname{grad} w=s_{\kappa} \circ r_{q}=0$. Then by (32) and (36) we have

$$
e^{-\Phi} \operatorname{Hess}\left(e^{\Phi} w\right)(v, v)=\operatorname{Hess} w(v, v) \geq|v|^{2}>0
$$

when $v \neq 0$. Hence $e^{\Phi} w$ is a convex function on $B_{R}(p)$ for any $R<\frac{1}{2 \sqrt{\kappa}}$, hence for $R^{\prime}$ where $R<R^{\prime}<\frac{1}{2 \sqrt{\kappa}}$. Therefore, $e^{\Phi} w$ is convex on $\overline{B_{R}(p)}$. Let 
$\lambda$ be the infimum of the smallest eigenvalues of $\operatorname{Hess}\left(e^{\Phi} w\right)$ on $\overline{B_{R}(p)}$. Then $\operatorname{Hess}\left(e^{\Phi} w\right)(v, v) \geq \lambda|v|^{2}$. Apparently, $\lambda>0$ because of the compactness of

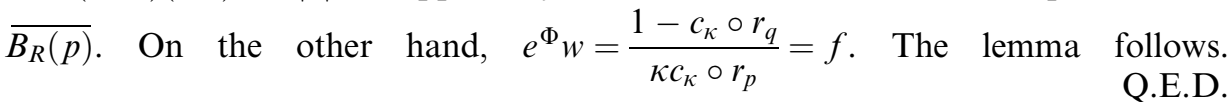

\section{REFERENCES}

[1] J. M. Bony, Principe du maximum, inégalité de Harnack et unicité du problème de Cauchy pour les opérateurs elliptiques dégénérés, Ann. Inst. Fourier 19 (1969), 227-304.

[2] G. CiтTI, Wiener estimates at boundary points for Hörmander's operators, Bollettino U.M.I., B. (7) 2 (1988), 667-681.

[ 3 ] D. Danielli, Regularity at the boundary for solutions of nonlinear subelliptic equations, Ind. Univ. Math. J. 44 (1995), 269-286.

[4] J. Eells \& J. SAmpson, Harmonic mappings of Riemannian manifolds, Amer. J. Math. 85 (1964), 109-160.

[5] P. Hajlasz and P. Strzelecki, Subelliptic p-harmonic maps into spheres and the ghost of Hardy spaces, Math. Ann. 312 (1998), 341-362.

[6] S. Hildebrandt, Harmonic mappings of Riemannian manifolds, LNM 1161, 1984, 1-117.

[7] J. Jost And C. J. Xu, Subelliptic harmonic maps, Trans. AMS. 350 (1998), 4633-4649.

[8] W. S. Kendall, Probability, convexity, and harmonic maps with small image I: uniqueness and fine existence, Proc. London Math. Soc. (3) 61 (1990), 371-406.

[9] R. S. Stricharts, Sub-Riemannian geometry, J. Diff. Geom. 24 (1986), 221-263.

[10] C. WANG, Subelliptic harmonic maps from Carnot groups, Calc. Var. 18 (2003), 95-115.

[11] Z. R. Zhou, Uniqueness of subelliptic harmonic maps, Ann. of Global Anal. and Geom. 17 (1999), 581-594.

Department of Mathematics

Central China Normal University

430079 WUHAN

P. R. CHINA

E-mail: zrzhou@mail.ccnu.edu.cn 